\title{
Towards better DNA chip hybridization using chaotic advection
}

\author{
Florence Raynal, Aurélien Beuf, Frédéric Plaza, Julian Scott, and Philippe Carrière \\ LMFA, CNRS-Université de Lyon, France; École Centrale de Lyon-Université Lyon 1-INSA Lyon; \\ ECL, 36 Avenue Guy de Collongue, 69134 Écully cédex, France
}

Michel Cabrera, Jean-Pierre Cloarec, and Éliane Souteyrand ${ }^{\mathrm{a})}$

INL (LÉOM, UMR CNRS 5512); École Centrale de Lyon, 69134 Écully cédex, France

(Received 16 July 2006; accepted 4 December 2006; published online 17 January 2007)

\begin{abstract}
Numerical studies for two protocols of micromixing based on chaotic advection to improve DNA chip hybridization are presented. The first protocol uses syringes; the other one, pumps. For both protocols, numerical Poincaré sections and Lyapunov exponents of the three-dimensional, time-periodic flow are investigated as functions of the period. Model experiments also confirm numerical results. Homogeneity of the dispersion of particles inside the chamber is of primary importance to achieve best chip reliability: although global chaos was obtained for both protocols, we find that the one employing the pumps is more likely to achieve better and more rapid hybridization. () 2007 American Institute of Physics. [DOI: 10.1063/1.2431322]
\end{abstract}

\section{INTRODUCTION}

The DNA chip has become one of the core technologies for genetic research purposes. ${ }^{1}$ Whereas, up to now, it has been mainly used for gene expression studies, this technology is now finding applications in the field of medical diagnosis. For obvious reasons, this is subject to its ability to provide highly reliable and rapid results while also allowing standard, automated operations. An important field of application is the detection of the presence (and abundance) of pathogenic viruses or bacteria. In that case, because very low concentrations may be involved, the detection method is required to be highly sensitive.

A DNA chip is composed of an array of biological probes fixed to a solid surface. A probe is a chain of nucleotides (i.e., cDNA, oligonucleotide) with a predetermined (synthetic) sequence of the four bases: $A, T, C$, and $G$ (the usual notation for adenine, guanine, cytosine, and thymine). Typically, the length of a synthetic sequence is in the range 12-60 bases. The array consists of spots, each of which contains many identical sequences characteristic of a particular genome or a particular mutation inside a genome; the exact number of sequences depends on the technology employed in making the chip, but, as an illustration, $10^{8}$ identical sequences for a spot of 200- $\mu \mathrm{m}$-diam is typical. With improvements in miniaturization techniques, it is possible to have hundreds of thousands of spots per $\mathrm{cm}^{2}$ (Refs. 2 and 3) (the order of magnitude of a chip surface area), allowing as many different sequences. The working principle of DNA microarrays is the so-called base-pairing or hybridization phenomenon. The situation is sketched in Fig. 1. Two single-stranded DNA fragments tend naturally to combine if their sequences are complementary to one another; that is, if $A-T$ or $G-C$ couples are formed at each location of the double-stranded molecule arising from assembling the fragments. Otherwise, the two fragments do not combine. Thus, when the array is

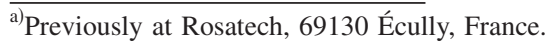

exposed to a solution containing single-stranded DNA samples (the targets), the latter have a tendency to hybridize with the complementary probe on the chip, if present. Since the targets have been marked with a fluorescent marker (or radioactive label) as a preliminary step, once the solution is blown out, only hybridized spots of the array fluoresce (or are radioactive), allowing determination of the chemical composition (the genotyping) of the DNA samples.

Hybridization is thus a critical step, even if many other steps, including the manufacture of the chip itself, are also important. The simplest way to perform hybridization, usually referred to as passive or static hybridization, is to put a drop of solution (50 $\mu \mathrm{l}$, for example) onto the DNA chip, cover it with a glass plate, and wait a certain amount of time. Usually, overnight hybridization is necessary to obtain meaningful results; furthermore, even after waiting that long, significant variations in the response of the chip are commonly observed. ${ }^{4}$ There is thus a need to speed up the hybridization process as well as improve the reliability of the results. Also, for near-future applications, the goal is to improve sensitivity so as to allow solutions with very low target concentrations. Theoretical studies of passive DNA chip hybridization usually employ a model, such as a Langmuir adsorption model, ${ }^{5,6}$ which describes the overall kinetics of the interaction between the chip as a whole and the solution as a whole. But in fact, hybridization in DNA chips involves at least two mechanisms: (i) diffusion of target molecules within the solution, a random process which ensures that targets reach the probes, and (ii) hybridization reactions between target and probe molecules (formation of hydrogen bonds between complementary bases). This interaction between the probe and the target is a very complicated process, dependent on many parameters, e.g., the length and complexity of the molecules and the temperature, and characterization of the resulting kinetics is beyond the scope of the present paper.

Hybridization at the surface of each spot removes target molecules from solution in the immediate, microscopic vicinity of the surface. Diffusion is needed to bring in fresh 


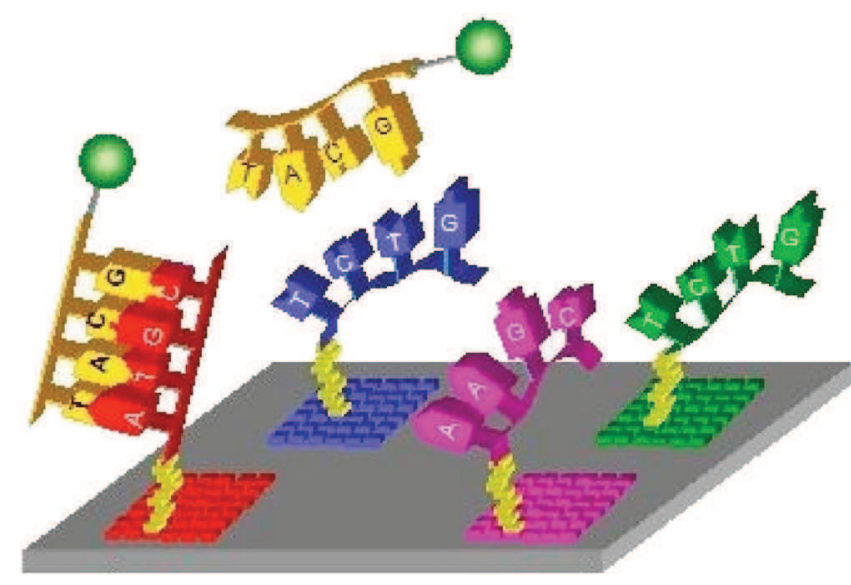

FIG. 1. Schematic view of a DNA chip. The targets hybridize to their complementary probes.

target molecules for reaction, taking a time of order $d^{2} / D$ for a molecule initially at distance $d$, where $D$ is the diffusion coefficient of target molecules. For water solutions, $D$ is typically of order $10^{-9}-10^{-10} \mathrm{~m}^{2} \mathrm{~s}^{-1}$ at most, ${ }^{7}$ whereas the solution layer is typically of vertical thickness $h \approx 500 \mu \mathrm{m}$ and horizontal extent $\ell \approx 10 \mathrm{~mm}$. The corresponding diffusion times are

$$
\tau_{\mathrm{h}}=\frac{h^{2}}{D} \approx 4 \text { to } 40 \mathrm{~min}
$$

and

$$
\tau_{\ell}=\frac{\ell^{2}}{D} \approx 30 \text { to } 300 \mathrm{~h} .
$$

Whether or not target molecules need to be brought in from such distances depends on their concentration and the number required for an observable result of hybridization. This is particularly important given the current aim of going to lower concentrations. Ideally, all target molecules in the solution should have the potential to react at all spots, which requires a long time of order $\tau_{\ell}$ if one relies on diffusion alone. Another aspect of the problem arises if the concentration of target molecules is initially nonuniform, i.e., if insufficient mixing has been applied prior to hybridization. In that case, diffusion is needed to render the concentration uniform, otherwise the results will vary depending on the initial concentration distribution in a potentially uncontrolled manner. Indeed, the large observed variability in spot hybridization between nominally identical tests may be a consequence of a poorly mixed solution.

Fluid flow applied during hybridization has the potential to resolve the problems described above, leading to the idea of active or dynamic hybridization. In addition to diffusion, target molecules are transported by the flow, which can greatly reduce the time required to sample all spots within the chip and render the target molecule concentration uniform by mixing. However, in small devices such as these, only creeping flow is possible; in particular, turbulence does not occur. Laminar flows are not intrinsically efficient mixing systems apart from chaotic advection flows ${ }^{8-10}$ and, even if the flow induces chaotic particle trajectories, attention needs to be paid to the topological properties of those trajectories. Indeed, in a chaotic flow, depending on the governing parameters, regular regions may coexist with chaotic ones, resulting in inhomogeneous concentration. Since the hybridization reaction depends on concentration, this may lead to variations in the response of the chip.

We recently proposed a chaotic stirring protocol adapted to the special geometry of DNA chip hybridization chambers. ${ }^{11}$ A fluid mechanical analysis was carried out, illustrating the important role of the numerical simulations to help identify the parameter ranges corresponding to best chip specificity. The system we investigated is a variant of the pulsed source-sink system first proposed by Aref and Jones $^{12}$ to achieve chaotic mixing in a two-dimensional (2D) flow of inviscid fluid. A practical consideration of such a pulsed mixing in a Hele-Shaw cell was later done by Evans et al. $^{13}$ Independently of our work, another team also proposed a chaotic stirring protocol for DNA chips, using pulsed pairs of syringe-driven source-sink systems: Stremler et al. carried out a 2D mathematical analysis of their flow, ${ }^{14,15}$ Cola et al. studied the experimental device, ${ }^{16}$ and McQuain et al. ${ }^{17}$ showed experimentally that hybridization was significantly improved by this apparatus. Note that not only chaotic mixing has been studied in the context of DNA chip analysis and some systems using nonchaotic fluid motion have also been proposed in the literature: Adey et al. ${ }^{18}$ proposed a system composed of a disposable flexible lid, which contains two air-driven bladders that continuously mix the hybridization fluid. Liu et al. ${ }^{19}$ proposed a micromixing technique based on cavitation microstreaming. Both papers showed hybridization signal enhancement with significantly improved signal uniformity.

In the present paper, we propose a variant (henceforth referred to as protocol B) of our previous chaotic stirring system (protocol A) and perform a comparison between these two protocols. Protocol A, proposed in our previous paper, is based on time-periodic injection/extraction of fluid in the hybridization chamber using a four-syringe system. Protocol B uses two pumps, each one alternatively inducing motion of the fluid in a closed loop formed by the hybridization chamber and an outer pipe. Using numerical simulations, we show that the second protocol can greatly enhance chip hybridization: better mixing, with less total volume and in less total time.

\section{THE MIXING DEVICE}

\section{A. The hybridization chamber}

Without entering into details of the microfluidic mixing device, some characteristics dictated by manufacturing constraints should be emphasized. First of all, with a view to automated processing of DNA samples, the manual coverslip system is replaced by a hybridization chamber, which consists of a large aspect-ratio cavity with fluid inlet/outlet ports located at the corners of the cavity. The width of the cavity is imposed by the size of the DNA chip, which we here consider to be a square of side $10 \mathrm{~mm}$. As shown in Fig. 2, the DNA chip (symbolized by the square on the lower wall of the cavity) is located in the central region, away from the 

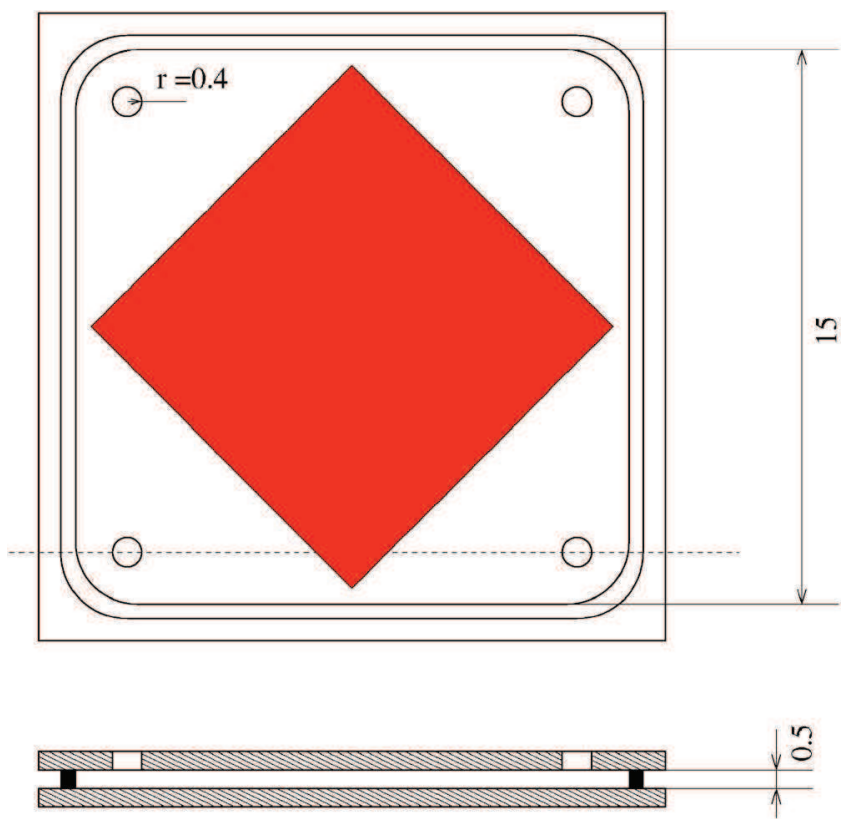

FIG. 2. Schematic view of the hybridization chamber. All lengths in mm. The DNA chip is symbolized by the central square.

four injection holes. For simplicity, we take the cavity to be also of square shape (except near its corners where a circular shape mimics the presence of an "O" ring) with width $L=15 \mathrm{~mm}$ and height $h=0.5 \mathrm{~mm}$. With such values, the volume of the chamber is

$$
V_{c h}=L \times L \times h \approx 110 \mu \mathrm{l} .
$$

Another parameter of importance which strongly depends on the available technology is the flow rate across the chamber. Fluid flow inside the chamber is induced by imposing a pressure difference between two opposite holes, connected to pipes in both protocols. During a given injection/ extraction phase, the flow is stationary, with a flow rate

$$
q=20 \mu \mathrm{s} \mathrm{s}^{-1},
$$

which can typically be obtained with syringe pumps or commercial peristaltic pumps. With such a value, the resulting Reynolds number $R_{\mathrm{i}}$ in the injection pipes of diameter $d=0.8 \mathrm{~mm}$ is

$$
R_{\mathrm{i}}=\frac{4 q}{\pi d \nu} \approx 30
$$

where the kinematic viscosity $\nu=10^{-6} \mathrm{~m}^{2} \mathrm{~s}^{-1}$ for pure water. For the hybridization chamber, we define a Reynolds number $R_{\mathrm{t}}$ based on the velocity scale $U_{\mathrm{t}}=q / \sqrt{2} h L$ and length scale $h$,

$$
R_{\mathrm{t}}=\frac{q}{\sqrt{2} L \nu} \approx 0.9
$$

In the pipes, away from the ends, the flow is of Poiseuille type and is therefore a solution of the Stokes equations. The same is true for the Hele-Shaw flow in the chamber, away

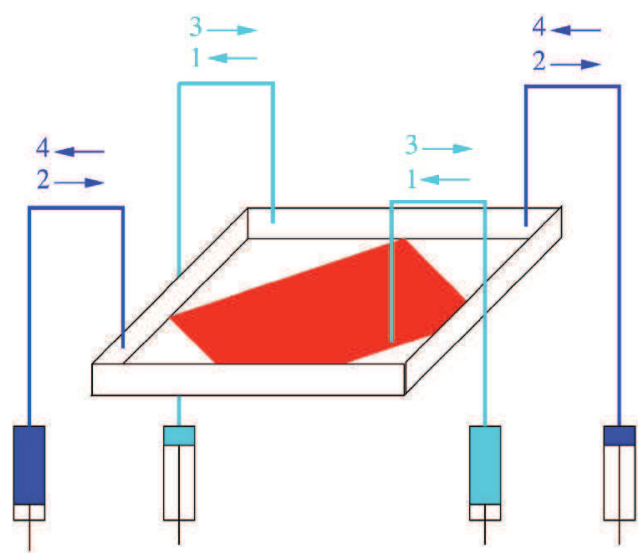

FIG. 3. Alternating injection scheme for protocol A. Each quarter-period step of the protocol involves two opposite syringes (the light-colored ones in steps 1 and 3, the dark ones in steps 2 and 4), the two other ones being inactive.

from the injection/extraction holes. Thus, it is not unreasonable to use Stokes flow as a first approximation to the velocity field.

\section{B. Stirring protocols}

Because the flow is almost everywhere of the Hele-Shaw type in the chamber, it can be considered as two dimensional to a first approximation. In 2D flows, chaotic trajectories can only be obtained if the flow is unsteady; hence we consider two time-periodic protocols. Note here that, since the chamber has square geometry, the flow field produced during an injection-extraction phase is symmetric about the line connecting two opposite holes. This property has also been used in the numerical calculations of the flow, as we will see later.

It must be yet underlined that, since the flow is essentially of the Hele-Shaw type inside the hybridization chamber, the problem is unchanged if the nondimensional number

$$
\frac{q T}{L^{2} h}
$$

is kept constant. In this way, our results can be interpreted in a more general context and, more particularly, can be applied to smaller total volumes of fluid.

\section{Protocol A}

The first protocol, referred to as protocol A, was described in Raynal et al. ${ }^{11}$ It can be thought of as the simplest such chaotic protocol for this kind of geometry and is based on alternating injections using two pairs of syringes, as shown in Fig. 3.

At the end of a quarter period, two syringes are full, the two others are empty. During an injection-extraction phase, the volume that enters the "sink" syringe is $v=q T / 4$. Therefore, the total volume necessary is 


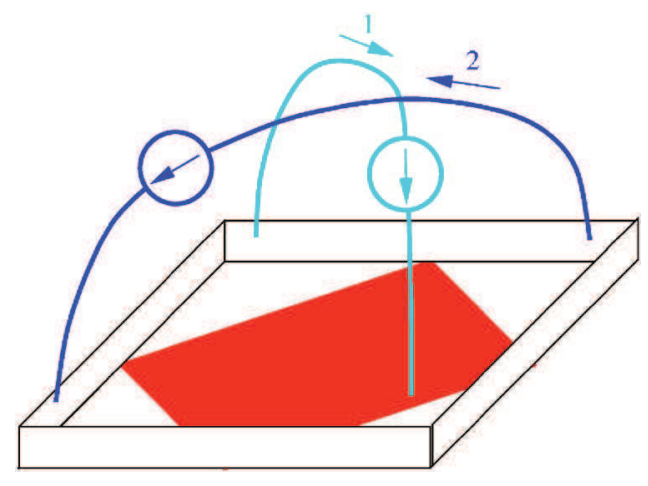

FIG. 4. Time-periodic scheme for protocol B. Each half-period step of the protocol involves one of the pumps, the other one being inactive. One pump always pushes the fluid in the same direction, or else is inactive.

$$
\begin{aligned}
V_{t o t} & =V_{c h}+2 v+v_{\text {pipe }} \\
& =V_{c h}+q T / 2+v_{\text {pipe }},
\end{aligned}
$$

where $v_{\text {pipe }}$ is the volume inside the pipes that connect each hole to each syringe. It is important to note that the total volume necessary for that protocol depends on the period $T$. However, $T$ cannot be arbitrarily chosen, because the stirring protocol has to be optimized: since $q$ is fixed, the only way to achieve this is by letting the period $T$ vary, and choosing the period that provides the best mixing within the chamber. Using Eq. (7), one can see that, once the best period is chosen, the volume inside the syringes is always the same fraction of the volume of the chamber, even if $h$ or $L$ is decreased. It is therefore important to check that this extra volume is not too large compared to the volume of the chamber.

It can be shown that the equations of the fluid-particle trajectories are invariant under the change of variables $t \rightarrow-t, \quad x \rightarrow-x, \quad y \rightarrow y$, and $z \rightarrow z$. This implies that the Poincaré sections $P_{t=0}$, corresponding to the position of particles at successive periods, starting at $t=0$, are invariant under reflection in the plane $x=0$. Moreover, a translation in time $t \rightarrow t+T / 4$ leaves the flow unchanged after rotation of angle $-\pi / 4$ (see Fig. 3). Thus, the Poincaré section $P_{t=T / 4}$ is obtained from the Poincaré section $P_{t=0}$ by a rotation of angle $-\pi / 4$. This property is important for chip hybridization, as we will see later.

\section{Protocol B}

The second protocol does not require the use of syringes, instead using two pumps. The pipes joining two opposite holes include a pump, as shown in Fig. 4. The stirring protocol is made time periodic by switching from one pump to the other at each half-period step. As for protocol A, the stirring process has to be optimized by choosing the best value for the period $T$. The total volume for this second protocol is

$$
V_{\text {tot }}=V_{c h}+v_{\text {pipe }}+v_{\text {pump }},
$$

where $v_{\text {pump }}$ is the pump volume. Note that, unlike protocol A, $V_{\text {tot }}$ does not depend on the period $T$. Indeed, the only extra volume for protocol $\mathrm{B}$ is that of the pipes and pumps. In our case the pump is peristaltic and so, as in most existing microfluidic applications, the pump volume is zero. We suppose that this extra volume is negligible.

It can be shown that the equations of the trajectories are invariant under the change of variables $t \rightarrow-t, x \rightarrow x$, $y \rightarrow-y$, and $z \rightarrow z$. This implies that the Poincaré sections $P_{t=0}$ for protocol B are invariant with respect to reflection in the plane $y=0$. Moreover, a translation in time $t \rightarrow t+T / 2$ leaves the flow unchanged after reflection in the plane $x=0$ (see Fig. 4). This implies that the Poincaré section $P_{t=T / 2}$ is obtained from the Poincaré section $P_{t=0}$ by reflection in $x$.

\section{Remark}

In the case of an ideal Stokes flow with no diffusion, we might wonder whether a particle should not always remain at the same depth in the domain. Indeed, numerical calculations for the velocity field show that the velocity can be everywhere considered as horizontal, except in the region of the source and sink, where particles undergo significant changes in their vertical positions. It is found that the volume of fluid in which the velocity field is not horizontal is about oneeighth of the total volume of the chamber (1/16 around each hole). When an injection/extraction phase is stopped, a fluid particle located in this volume was either going up or down, but will remain at the same depth at which it had stopped when a new injection/extraction phase begins, since it is now located far from the source and sink. This allows for oneeighth of the volume of the chamber to change vertical position at each new injection/extraction phase; that is, four times in a period for protocol A, and twice for protocol B. One must bear in mind however that for the first globally chaotic case found for protocol A $(T=10 \mathrm{~s})$, the total volume is almost twice the volume of the chamber, ${ }^{11}$ so that, in fact, a fluid particle will change horizontal plane at the same rate for both protocols.

\section{Modeling the device}

The numerical scheme and experimental setup for protocol A have already been presented in the previous paper. ${ }^{11}$ Here we give a brief description, with a few more details concerning protocol $\mathrm{B}$.

\section{Numerical scheme}

Numerical calculations have been performed to determine the fluid particle trajectories generated by the alternated injection system. The velocity field, considered as steady during each phase, was obtained by solving the Stokes equations: a discrete velocity field $\boldsymbol{U}_{h}$ was obtained using a finite element method with quadratic approximation of the velocity (the so-called $P_{1}-P_{2}$ or Taylor-Hood element, with linear approximation of the pressure; see Ref. 20). The finite element grid shown in Fig. 5, which describes half the chamber and part of the pipes, contains 33849 vertices and 214304 segments, and consequently, 248153 grid points, per component of the velocity. The use of such a fine grid is dictated by the accuracy required when using the Lagrangian tracking method, which strongly depends on satisfaction of the continuity constraint. Numerically, the flow field inside the chamber is imposed by prescribing the Poiseuille profile at the 


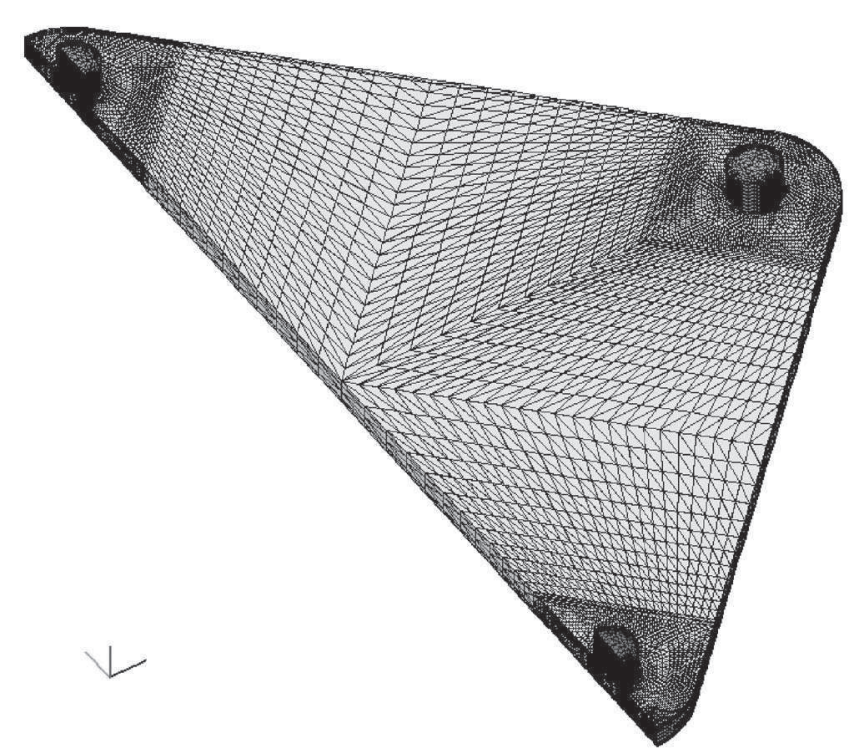

FIG. 5. Finite element grid of the half chamber, used for the numerical simulations.

entry pipe; constant pressure and Neumann conditions on the velocity are prescribed at the outlet. No-slip conditions are prescribed elsewhere except the vertical symmetry boundary. For protocol A, the syringes and pipes are modeled by four "long enough" straight pipes; the flow was determined numerically at the entry of each pipe, until the flow inside the pipe is reasonably established. It is modeled by a Poiseuille flow thereafter. The case of protocol B is quite similar, although in that case the pipes are bent so as to have a semicircular shape above the chamber. The flow field is completed afterward by symmetry in order to track the particles in the entire domain. The full flow field is thus achieved by a $\pi / 2$ rotation at each $T / 4$ for protocol A, or by a $\pi / 2$ rotation followed by a $-\pi / 2$ rotation, each $T / 2$, for protocol $\mathrm{B}$. The details of the Lagrangian tracking method and the associated Lyaponov exponent calculation method are given in a separate paper. ${ }^{21}$ In short, trajectories are obtained using a standard fourth-order Runge-Kutta integration adapted to piecewise defined functions while Lyapunov exponents are determined using a corresponding third-order time scheme applied to the Jacobian method. ${ }^{22}$

\section{Scaled-up experimental setup}

As for protocol A, experimental investigations for protocol B were performed with a 10:1 scaled-up model. A 2-mm-thick horizontal green laser sheet (wavelength $532 \mathrm{~nm}$ ) was created using a divergent lens, in order to induce rhodamine B fluorescence. The bottom of the chamber was made of black ertacetal ${ }^{\circledR}$, in order to prevent water and rhodamine adsorption and laser reflection, while the sides and top were made of glass. A drop of highly concentrated rhodamine solution was injected in one of the external pipes, near the initial fluid outlet. The alternated injection was then started and top-view photographs recorded after each period.

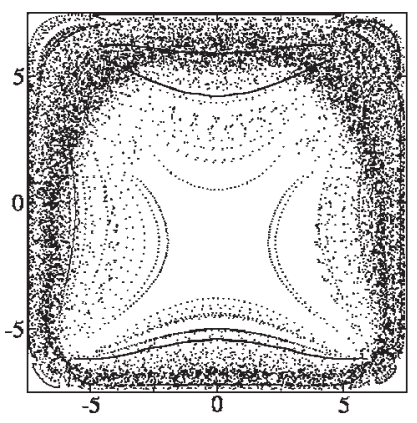

(a)

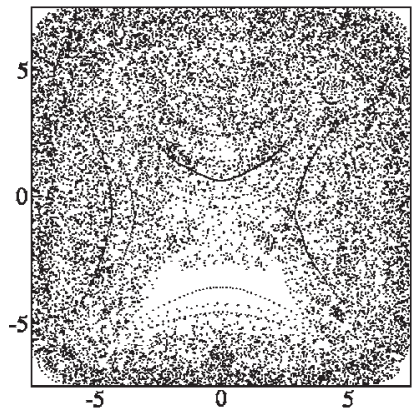

(c)

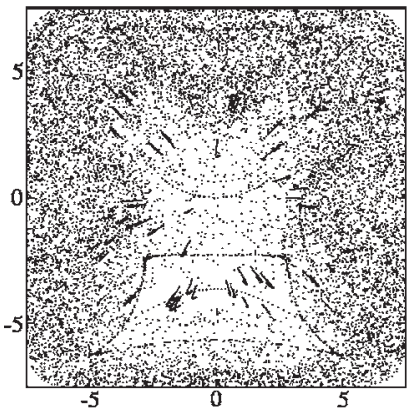

(e)

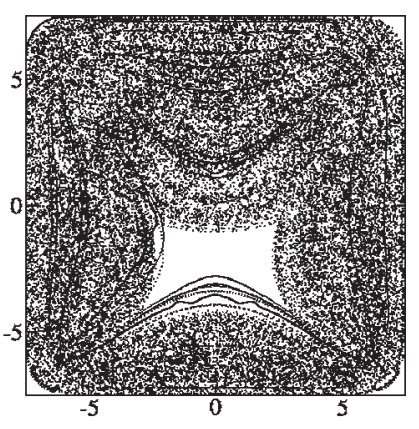

(b)

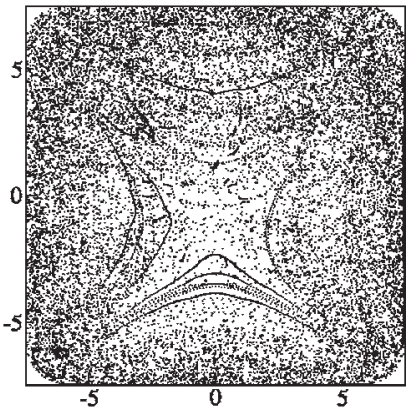

(d)

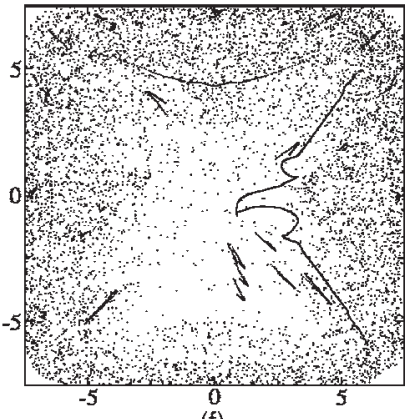

(f)
FIG. 6. Top view of three-dimensional Poincaré sections for protocol A: (a) $T=2 \mathrm{~s}$; (b) $T=4 \mathrm{~s}$; (c) $T=6 \mathrm{~s}$; (d) $T=8 \mathrm{~s}$; (e) $T=10 \mathrm{~s}$; (f) $T=12 \mathrm{~s}$.

Reynolds similarity imposes a flow rate of $11.2 \mathrm{ml} \mathrm{m}^{-1}$. Thus, a ratio of $9.5 \times 10^{-3}$ is found between the real period $T$ and the scaled model period $\underline{T}$.

For sake of simplicity, we used only one experimental setup analogous to Fig. 4, with two reversible peristaltic pumps for both protocols. Indeed, protocol A can be modeled with such an experimental setup, provided that the pipes are long enough: the fluid that enters a sink must never reach the pump, to avoid mixing in the pump. Note however that, since the volume extracted is proportional to the period $T$, the larger the period, the greater the volume in the pipes. We used pipes with two different internal diameters for the two protocols: the larger one, $d=5.5 \mathrm{~mm}$, for protocol $\mathrm{A}$, in order to avoid pipes which are too long; the other one, $d=3 \mathrm{~mm}$, for protocol B. We found no significant difference in the visualizations using the pumps or the syringes.

\section{RESULTS AND DISCUSSION}

\section{A. Results}

We present now top-view Poincaré sections of the flow, together with Lyapunov exponents for different periods of 


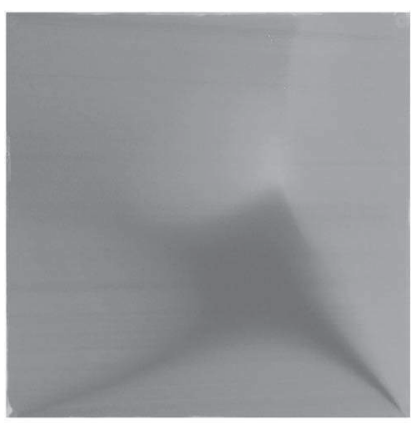

(a)

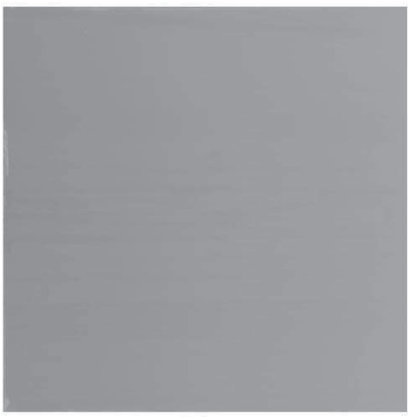

(c)

FIG. 7. Top-view flow visualization (rhodamine fluorescence) for protocol A after six periods of the flow field: (a) $T=4 \mathrm{~s}(\underline{T}=420 \mathrm{~s})$; (b) $T=8 \mathrm{~s}$; (c) $T=10 \mathrm{~s}$, and after three periods: (d) $T=16 \mathrm{~s}$.

the flow field. The experiments are compared with the numerical calculations. Top-view Poincaré sections of protocol A are shown in Fig. 6 for six periods, $T=2, T=4, T=6$, $T=8, T=10$, and $T=12 \mathrm{~s}$. We already showed such results for protocol A (Ref. 11): we had searched for the existence of regular regions and concluded that a completely chaotic flow was obtained for $T \geq 10 \mathrm{~s}$. In the Poincaré sections shown in Fig. 6, there is only one trajectory, with initial condition taken in the chaotic region, calculated for very long times. The reader is cautioned that, since we deal with a timeperiodic, three-dimensional (3D) flow, the Poincaré section cumulates the successive positions of a fluid particle after each period, and is therefore a set of points in 3D space. Indeed, in Fig. 6(d) $(T=8 \mathrm{~s})$, the regular region is hidden by positions of the fluid particle above or below this region. As a consequence, in the experiment where we cannot neglect the effect of molecular diffusion, we can expect that this region slowly disappears with time, due to vertical mixing. Top-view photographs of the experiment for protocol A are shown in Fig. 7 for four different periods, $\underline{T}=420 \mathrm{~s}$ (corresponding to a period of $T=4 \mathrm{~s}$ at the real scale), $\underline{T}=840 \mathrm{~s}$ $(T=8 \mathrm{~s})$, and $T=1050 \mathrm{~s}(T=10 \mathrm{~s})$ after six periods of the flow field, and also $\underline{T}=1680 \mathrm{~s}$ ( $T=16 \mathrm{~s}$ ) after three periods of the flow field [same total time as in Fig. 7(b), $T=8 \mathrm{~s}$ ]. The dye indeed appears to be well mixed for $T=10 \mathrm{~s}$ and for all higher periods tested (up to $T=16 \mathrm{~s}$ ). For $T=4 \mathrm{~s}$ and $T=8 \mathrm{~s}$, the unmixed region is similar to the regular region shown in the Poincaré sections of our previous paper. When letting the experiment go, we find that the unmixed region is quite ro-

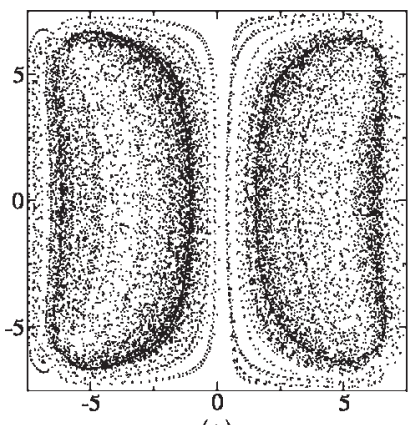

(a)

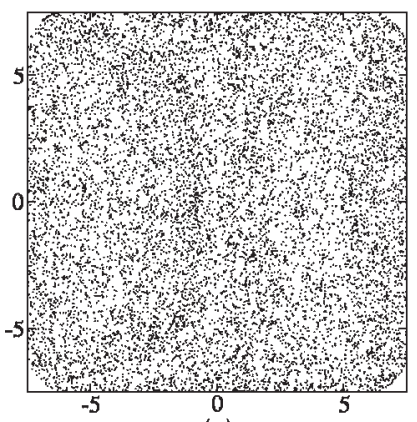

(c)

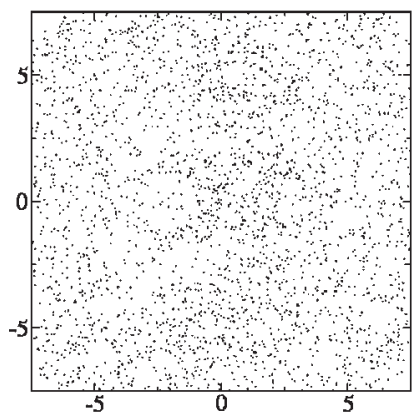

(e)

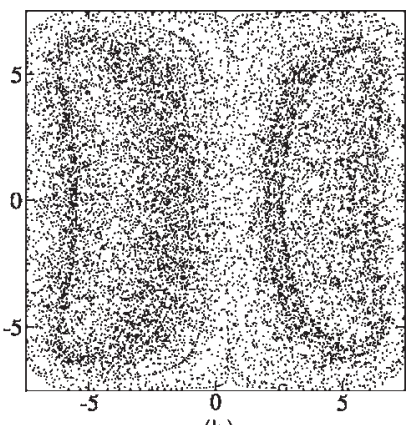

(b)

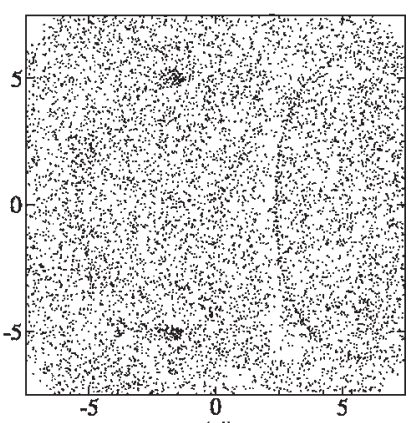

(d)

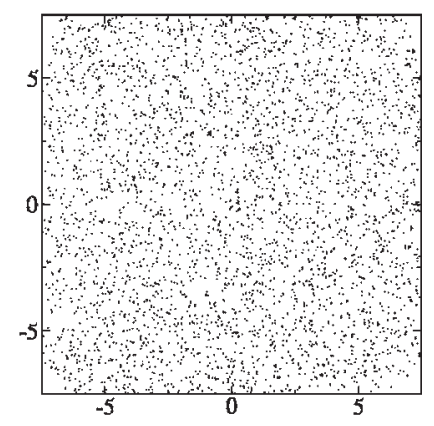

(f)
FIG. 8. Top view of three-dimensional Poincaré sections for protocol B: (a) $T=0.5 \mathrm{~s}$; (b) $T=1 \mathrm{~s}$; (c) $T=2 \mathrm{~s}$; (d) $T=4 \mathrm{~s}$; (e) $T=6 \mathrm{~s}$; (f) $T=8 \mathrm{~s}$.

bust for the period $T=4 \mathrm{~s}$, while it tends to disappear slowly with time for $T=8 \mathrm{~s}$; this is in total agreement with the Poincaré sections shown here.

In Fig. 8 we show Poincaré sections for protocol B for six periods, $T=0.5, T=1, T=2, T=4, T=6$, and $T=8 \mathrm{~s}$. No regular region exists for any of those periods, which implies that chaos is global even when the period is small. Therefore, we checked experimentally that the length (more especially the volume) of the pipes was not a crucial parameter for this protocol: using longer pipes just increases the total time for mixing (increasing the residence time inside the pipe), but does not alter the overall mixing inside the chamber. Indeed, the rhodamine was rapidly mixed for all periods tested: Figure 9 shows experimental results for two different periods, $\underline{T}=210 \mathrm{~s}(T=2 \mathrm{~s})$ and $\underline{T}=420 \mathrm{~s}(T=4 \mathrm{~s})$. In both cases, the rhodamine was dispersed after the same total time $(16 \mathrm{~s}$ in real time), although the concentration is still slightly inhomogeneous in Fig. 9(d).

Lyapunov exponents as a function of time are shown for protocols A and B, respectively, in Figs. 10 and 11. For periods $T=4$ and $T=8 \mathrm{~s}$ of protocol A, where chaos is not 


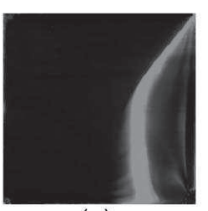

(a)

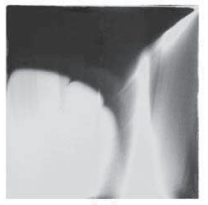

(e)

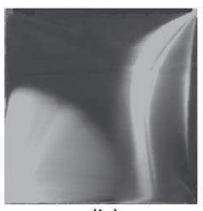

(b)

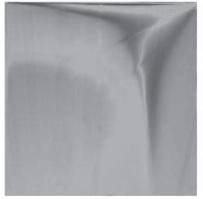

(f)

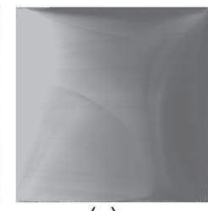

(c)

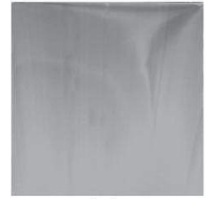

(g)

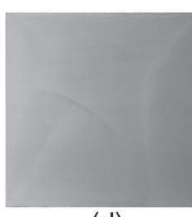

(d)

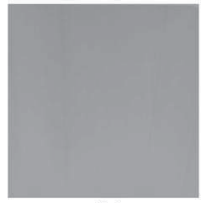

(h)
FIG. 9. Top-view flow visualization for protocol B for two different periods of the flow field, $T=2 \mathrm{~s}(T=210 \mathrm{~s})$ and $T=4 \mathrm{~s}(\underline{T}=420 \mathrm{~s})$. Top: $T=2 \mathrm{~s}$ at times (a) $t=T$, (b) $t=2 T=4 \mathrm{~s}$, (c) $t=4 T$, and (d) $t=8 T=16 \mathrm{~s}$. Bottom: $T=4 \mathrm{~s}$ at times (e) $t=T=4 \mathrm{~s}$, (f) $t=2 T$, (g) $t=3 T$, and (h) $t=4 T=16 \mathrm{~s}$.

global, the initial point was chosen to lie inside the chaotic region. The calculations were stopped after a specified number of periods and/or when the Lyapunov exponent had sufficiently converged, which explains why the curves do not all stop at the same time. Note that the Lyapunov exponents converge much more rapidly for protocol B. For the sake of clarity, the two figures are not shown with the same horizontal and vertical scales, but one must bear in mind that the periods shown for protocol B are smaller than those for protocol A.

All the results are summarized in Fig. 12, which shows the positive Lyapunov exponent after convergence of the calculations, for both protocols, as a function of the period of the flow. Note that more periods were involved than shown in the Poincaré sections. For each protocol, it is apparent that the Lyapunov exponents decrease with the period. It is even more interesting to note that for each period in common, the Lyapunov exponent for protocol $\mathrm{B}$ is higher than that of protocol A.

Recently, Stremler and $\mathrm{Cola}^{15}$ proposed using Kolmogorov-Sinai (KS) entropy ${ }^{23}$ and a 2D model of their mixing apparatus ${ }^{17}$ in an attempt to optimize their protocol.

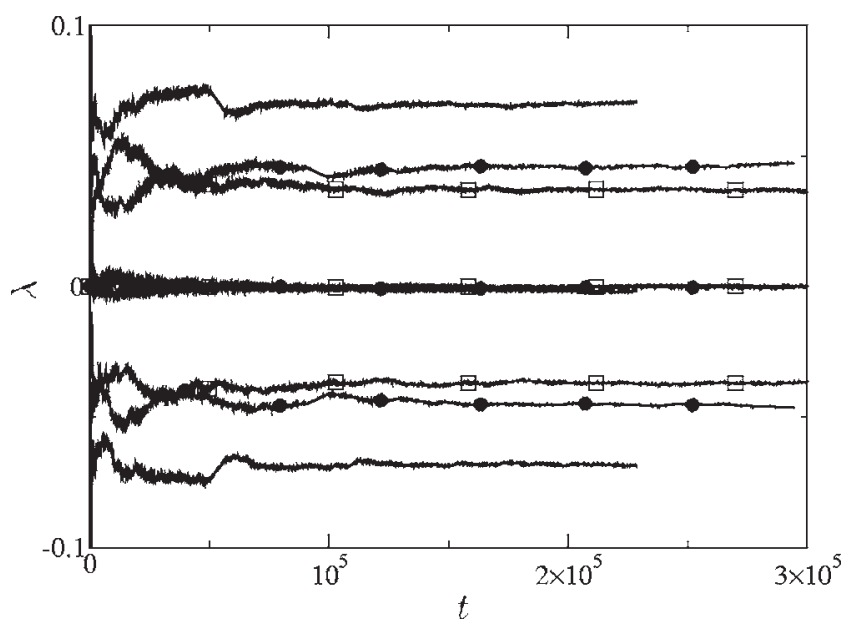

FIG. 10. Lyapunov exponents $\lambda$ for protocol A for three different periods, $T=4 \mathrm{~s}(-), T=8 \mathrm{~s}(\bullet)$, and $T=10 \mathrm{~s}(\square)$, as a function of time $t$ (in seconds).

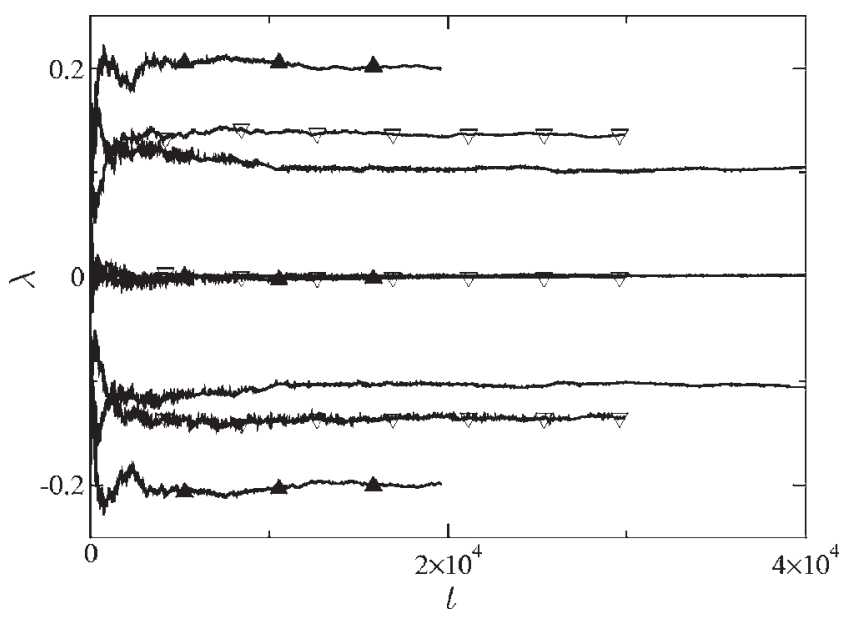

FIG. 11. Lyapunov exponents $\lambda$ for protocol B for three different periods, $T=1 \mathrm{~s}(\boldsymbol{\Delta}), T=2 \mathrm{~s}(\nabla)$, and $T=4 \mathrm{~s}(-)$, as a function of time $t$ (in seconds).

In $2 \mathrm{D}$ flows, the KS entropy was shown to be equal to the area of the chaotic region, multiplied by the Lyapunov exponent. If we suppose that our top-view Poincaré sections are two dimensional, using the KS entropy leads to the conclusion that protocol B is better (chaos is global whatever the period and the Lyapunov exponent is always higher for a given time period). However, judging 3D Poincaré sections with a $2 \mathrm{D}$ projection can be misleading. For instance, the existence of the regular region at the center of the chamber cannot be detected using a 2D projection. Also, two points that seem very close to each other on the top-view Poincaré section could lie at very different depths. In order to quantify the extent to which 3D Poincaré sections fill the volume, we should partition the volume into cubic elements. We need at least three cubes in the height (so that we can detect an empty zone at the center of the cavity, for instance) and, since $L=30^{*} h$, this implies at least $n=30^{2} \times 3^{3}=24300$ cubes. Thus, the percentage of the domain covered, $\mu$, is

$$
\mu=100 \times \frac{n^{\prime}}{n},
$$

where $n^{\prime}$ is the number of nonempty cubes (where at least one point of the Poincaré section lies). Of course, in order for the statistics to be reasonably converged, this requires at least ten more points than cubes, leading to unreasonably expen-

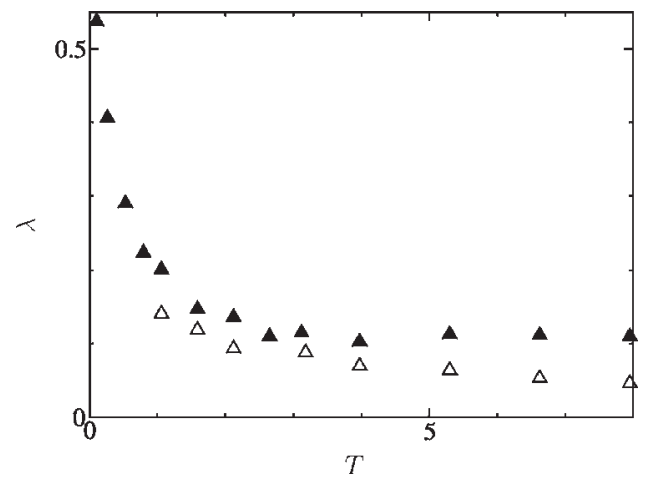

FIG. 12. Positive Lyapunov exponents $\lambda$ for protocols $A(\triangle)$ and $B(\boldsymbol{A})$ as a function of the period $T$ (in seconds). 


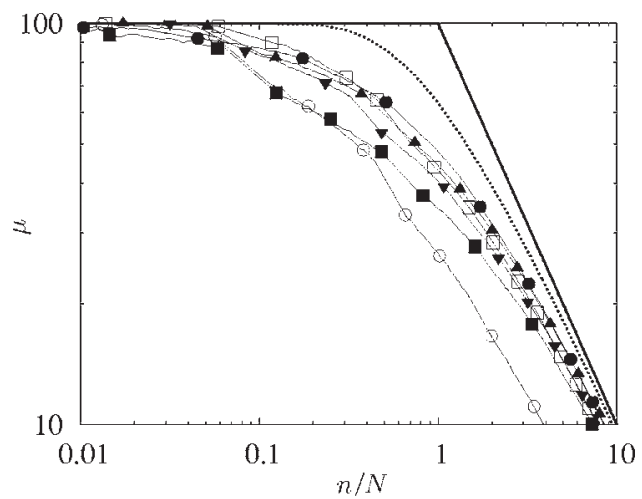

FIG. 13. Percentage of the domain covered, $\mu$, as a function of $n / N$, where $n$ is the number of cells, and $N$ is the total number of points of the Poincare section, for protocol A; dashed line: random distribution; solid line (without symbols): regular distribution; $T=2 \mathrm{~s}(\boldsymbol{\square}), T=4 \mathrm{~s}(\boldsymbol{\bullet}), T=6 \mathrm{~s}(\boldsymbol{\Delta}), T=8 \mathrm{~s}$ $(\boldsymbol{\nabla}), T=10 \mathrm{~s}(\square), T=12 \mathrm{~s}(\bigcirc)$.

sive calculations. Moreover, as emphasized in Ref. 21, there are particular problems associated with near-wall particles for which tracking is especially difficult, which tend to reduce the number of available points in the Poincaré section.

Aiming at a more quantitative approach, we propose the following:

(1) The cavity is divided into $n=n_{L}^{2} \times n_{h}$ parallelepipedic cells, where $n_{L}$ and $n_{h}$ are, respectively, the number of horizontal and vertical divisions. The cells are chosen nearly cubic, i.e., $n_{L}$ and $n_{h}$ are such that

$$
\frac{h}{n_{h}} \leq \frac{L}{n_{L}} \leq \frac{h}{n_{h}-1}
$$

The number of cells $n$ is varied from 100 to around $10^{6}$.

(2) For each $n$, the percentage of the domain covered, $\mu$, is calculated using formula (11).

(3) Since the number of points $N$ in each Poincaré section is different, the result is rendered independent of $N$ by plotting $\mu$ as a function of $n / N$.

The results for protocols $\mathrm{A}$ and $\mathrm{B}$ are shown on a log-log scale in Figs. 13 and 14, respectively. Note that the Poincaré sections of protocol A have many more points than those for protocol B; we verified that the curves in Fig. 13 were basically unchanged when considering only half of the points. The results are compared to the case of a random distribution of points inside the cavity (dashed line), which mimics the case of ideal chaos and provides a reference. The case of points distributed on a cubic lattice is also shown as a solid line: in that case, the percentage of the domain covered equals 100 as $n / N$ increases, up until there is one point per cell, then it decreases like $1 / n$. The solid symbols represent periods in the range $2 \mathrm{~s} \leq T \leq 8 \mathrm{~s}$, which appear for both protocols. Clearly, periods $T \geq 2 \mathrm{~s}$ in protocol $\mathrm{B}$ are the nearest to the random case.

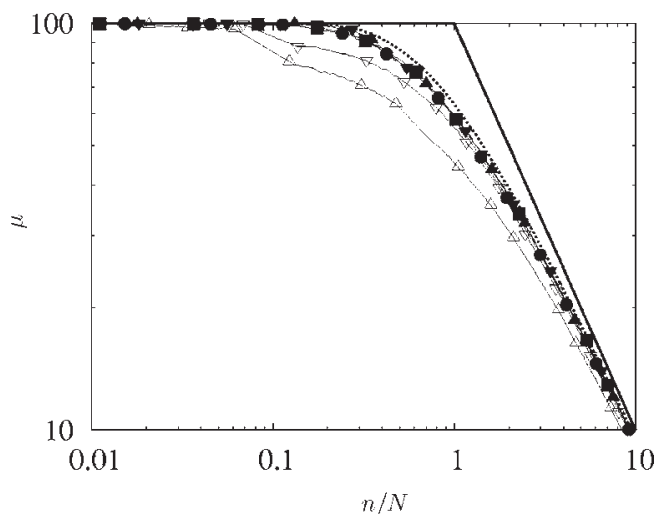

FIG. 14. Same as Fig. 13 for protocol B. $T=0.5 \mathrm{~s}(\triangle), T=1 \mathrm{~s}(\nabla), T=2 \mathrm{~s}$ $(\boldsymbol{\square}), T=4 \mathrm{~s}(\boldsymbol{\bullet}), T=6 \mathrm{~s}(\boldsymbol{\Delta}), T=8 \mathrm{~s}(\boldsymbol{\nabla})$.

\section{B. Discussion}

\section{Absence of regular islands}

Because the flow is unsteady, it is important to bear in mind that, for both protocols, any regular regions move with time. Thus, if every fixed region of space is visited by chaotic trajectories (or by labeled biological material in the real device), even though it may lie within a regular region for a while (without any marked particles), the presence of such regions would not be detected for high enough concentrations of labeled DNA. For protocol A, for instance, the Poincaré section $P_{t=T / 4}$ is obtained from $P_{t=0}$ after rotation of angle $-\pi / 4$. As a result, if the regular regions are not located at the center of the chamber, there will be no overlap of the position of the regular regions at different times, and one can assure that all fixed regions of space are visited by labeled biological macromolecules. This is the case, for instance, for the large regular region found both experimentally and numerically for the period $T=4 \mathrm{~s}$ and numerically for $T=6 \mathrm{~s}$, or for the smaller region which appears for $T=8 \mathrm{~s}$. For the case $T=2 \mathrm{~s}$, however, the large regular region overlaps the center of the chamber, which means that the central region is very unlikely to be visited.

For very small quantities of labeled macromolecules, however, the fact that some spots on the array are not visited for a while could lead to inhomogeneous hybridization, and consequently, to an incorrect reading of the chip. However, it is difficult to make sure that absolutely no regular region exists in the 3D flow. Consider, for instance, the case of protocol A and ideal Stokes-flow conditions, without diffusion, as in our numerical simulations: for a large enough period $T$, the particle located at the center of the chamber at $t=0$ is swallowed by an external pipe during the first injection/extraction phase. It remains there until the thirdquarter period, when the pipe acts as a source, reinjecting the particle into the chamber. In the ideal conditions described above, the particle returns exactly to its initial location at the end of the third injection/extraction phase, as indeed it does at the end of each multiple of $3 T / 4$. Thus, the center of the chamber is a period-3 periodic point of the Poincaré section. We calculated numerically the time needed for the central particle to enter sufficiently far into one of the external pipes, that it is stationary during the next quarter period: we found 
that this happens for periods exceeding $10.1 \mathrm{~s}$. For $T>10.1 \mathrm{~s}$, similar reasoning applies to neighboring particles, indicating the existence of a regular region for protocol A. From this point of view, of the periods tested for this protocol, $T=10 \mathrm{~s}$ appears to be the most efficient. However, because of the parabolic velocity profile, particles towards the top or bottom of the chamber have smaller velocities and do not follow the central particle into the pipe. Thus, the above regular region is limited in vertical extent, and is not visible in our 3D Poincaré sections, since particles outside the region can go above or below it. In the experiments, although a slice of fluid is visualized thanks to the laser sheet, we saw no sign of the existence of such a regular region (Fig. 7); this can be explained by the action of molecular diffusion, and by the fact that the Stokes approximation does not apply near the injection/extraction holes, as explained earlier, so that fluid particles in the experiment need not come back to their initial position when the flow is reversed.

The slow convergence in time of the Lyapunov exponents for protocol A could be related to the presence of regular regions, in the neighborhood of which particles remain "stuck" for a while before they come back into the chaotic bulk of the flow: we checked that this was the case for the two periods $T=4$ and $T=8 \mathrm{~s}$, where the particles remain for many periods near the large regular regions apparent in the Poincaré sections, but it is difficult to make sure that this is not also the case for the period $T=10 \mathrm{~s}$, where very small regular regions could exist and not be apparent from the top view of the corresponding Poincaré section. On the other hand, the very rapid convergence of the Lyapunov exponents for protocol B leads us to believe that no regular regions are present.

Another problem arises when a particle arrives very near the top or bottom wall: in the absence of diffusion, it remains at the same depth in the domain, providing it has moved sufficiently far from the holes. Because of the parabolic velocity profile, its velocity is very slow so that it does not move much during one period. Moreover, in the case of protocol A, the mean flow at one point during one period is zero, which means that a particle stuck near a wall remains even longer than for protocol $\mathrm{B}$, where there is a net horizontal flux from one side to the other. This again can explain the much slower convergence in time of the Lyapunov exponents for protocol A.

These results are also confirmed by Figs. 13 and 14. It is reasonable to suppose the statistics converged when the number of points $N$ inside the cavity is at least ten times more than the number of cells. It is apparent that the best domain coverage for protocol A is achieved for $T=10 \mathrm{~s}$. For protocol B, however, all periods $T \geq 2 \mathrm{~s}$ lead to $100 \%$ coverage for $n / N \leq 0.1$.

\section{Homogeneous chaos}

Although global chaos can be obtained for both protocols, it is also important that Poincaré sections be as homogeneous as possible, i.e., points are distributed regularly inside the whole volume. Only in this case can the probability density of the particle position be considered as homoge- neous over the chip surface, so that the chemical reaction takes place at any point on the chip with the same probability. For protocol A, and all cases with no obvious regular region in Fig. $6(T \geq 10 \mathrm{~s})$, the distribution of points is not completely homogeneous and the central region is less frequently visited than the periphery. This feature is much less pronounced for $T=10 \mathrm{~s}$ than for $T=12 \mathrm{~s}$ (or $T=16 \mathrm{~s}$ in our previous paper), however, so that the smaller period is again found to be more efficient. This is very probably related to the presence of the thin regular region at the center of the chamber for $T>10.1 \mathrm{~s}$, discussed earlier. For protocol B, the distribution of points seems much more homogeneous for all periods tested. However, the periods $T \leq 1$ s show a phenomenon described by Bajer and Moffat ${ }^{24}$ as "trans-adiabatic drift": a fluid particle migrates across the family of adiabatic surfaces, with trajectories that remain a long time near those of the steady case in which the two sinks and the two sources are run together.

These qualitative conclusions are confirmed by looking at Figs. 13 and 14: the curves corresponding to protocol $\mathrm{B}$ and periods $T \geq 2 \mathrm{~s}$ are the only ones lying very nearly on the curve representing a random distribution. Based on the criterion of homogeneity, protocol B is again more efficient than protocol A.

\section{Total volume small enough}

A biochemical reaction takes place on the chip when the labeled DNA molecules hybridize to their complementary probe. To a first approximation, the speed of this reaction can be taken to be proportional to the concentration in labeled DNA. It is thus important to have a total volume as small as possible, in order to have the highest concentration possible. For protocol A, global chaos was found for $T$ large enough. However, increasing the period also increases the volume of fluid inside the syringes, as shown by Eq. (9). For the first globally chaotic protocol found $(T=10 \mathrm{~s})$, the total volume (not taking into account the volume in the pipes) is almost twice the volume of the chamber. As a result, for a given quantity of labeled DNA, the concentration has been divided by two. For protocol B, the total volume is independent of the period, so that, if the volume of the pipes and pumps is small enough, the concentration of labeled DNA may be much larger than for protocol A.

\section{Rapid mixing}

Although the speed of reaction is proportional to the concentration, the total time for hybridization is primarily related to rapid mixing: the labeled particles, introduced at one point and time, must spread rapidly over the whole volume.

A classical way to measure mixing ability is via the Lyapunov exponents, which measure the exponential rate of separation of two nearby particles; it gives an idea of the rapidity of dispersion of particles inside the flow and can be considered as the inverse of a mixing time scale. Thus, the larger the Lyapunov exponent, the more rapid the mixing inside the chaotic region. It is interesting to note that, although the flow is $3 \mathrm{D}$ and time periodic, one of the three 
Lyapunov exponents is always nearly zero for each period of each protocol, as shown in Figs. 10 and 11. This confirms that chaos is mainly produced by the nonstationarity, like in purely two-dimensional flows; the vertical effects near the sink and the source just allow a fluid particle to change of horizontal plane in a reasonably small time. For a given protocol, as already explained, the Lyapunov exponent decreases with the period $T$. Therefore, mixing is more rapid for smaller periods of the flow field, and, from this point of view, the period should not be chosen too large. Note, however, once again, that for protocol B, the Lyapunov exponent is quite constant for $T \geq 3 \mathrm{~s}$. Also, for all periods in common, the positive Lyapunov exponent is always larger for protocol $\mathrm{B}$, so that the mixing time based on this parameter is smaller. This, again, favors the second protocol.

Just looking at some Poincaré section and judging which is the most homogeneous is not enough: even if the molecules are mixed after a few periods, the total time depends on how long the period is. This was pointed out by Raynal and Gence, ${ }^{25}$ in two-dimensional flows, who introduced a "flux" of chaotic particles (namely the size of a lobe from lobe dynamics ${ }^{26}$ divided by the period) and showed that this parameter was a maximum for a given period in their model flow. Indeed, for small values of the period, the flow is very close to the steady case and not globally chaotic (the size of the lobe is exponentially small). In a closed flow, however, the size of the lobes is bounded and the parameter goes to zero for large values of the period. Rom-Kedar and Poje ${ }^{27}$ showed later this result in a more general case. Although this result has been demonstrated for $2 \mathrm{D}$ flows, we expect quasi-2D Hele-Shaw flows, such as the present ones, to also have an optimum period for mixing rapidity. In other words, for two globally chaotic flows, the one which mixes more rapidly is that with the smallest period $T$, and protocol B seems more appropriate once again.

One could argue that the limiting factor for total time is the kinetics of the hybridization, rather than the mixing time. However, one must bear in mind that the labeled molecules are introduced in a very small volume (therefore highly concentrated) at the beginning of the process. If the spreading of molecules is not rapid enough, the spots of the array visited at the beginning, while the volume containing labeled molecules is still small and concentrated, will potentially be much more hybridized than the others. For this reason, the spreading of the molecules over the whole domain must be achieved at smaller time scales than the kinetics of hybridization. This is a classical result in chemical engineering for turbulent flows, where the micromixing time must be as small as possible when compared to the reaction time. And, for this reason again, protocol B appears much more efficient than protocol A.

\section{Miniaturizing the device}

We turn now to the feasibility of reducing the size of a device designed in the spirit of protocol B. As discussed earlier, such size reduction is desirable to allow as small DNA samples as possible. While the volume of the hybridization chamber itself can easily be reduced by decreasing its depth, the external dead volume must also be sufficiently small. For fluid mechanics engineers, the most important problem associated with miniaturization is a significant increase of the pressure drop across the microfluidic system. ${ }^{28}$ Here we consider a device using standard commercial micropumps that can presently be manufactured. Of course, future developments of miniature pump technology may well allow still further improvements, but our aim is to see what could currently potentially be achieved.

Let us begin with our model: in our numerical simulations, two holes were connected by a pipe of diameter $d=0.8 \mathrm{~mm}$ and length $15 \times \sqrt{2} \times \pi \simeq 67 \mathrm{~mm}$, implying a total external dead volume of $V_{\text {ext }}=2 \times 33 \mu$ l. In a scale $1: 1$ experiment corresponding to our calculations, connecting two opposite holes via the peristaltic pump would require a longer pipe, of maximum total length $\mathcal{L}=2 \times 200 \mathrm{~mm}$, for example. In order to keep the same external volume, the pipe's diameter would need to be $d=0.33 \mathrm{~mm}$. The Reynolds number in the injection pipe would then increase by a factor of up to 2.4 for the same flow rate [according to Eq. (5)] and the flow would remain laminar. The pressure drop in the pipes may be easily evaluated using the Poiseuille law,

$$
\delta P_{\mathrm{p}}=\mathcal{L} \times\left(\frac{128 \mu q}{\pi d^{4}}\right),
$$

where $\mu$ is the dynamical viscosity of water, leading to $\delta P_{\mathrm{p}} \approx 2.8 \times 10^{4} \mathrm{~Pa}$. The pressure drop inside the chamber can be estimated using the Hele-Shaw approximation as

$$
\delta P_{\mathrm{ch}} \propto \mu q / h^{3},
$$

where the numerical constant of proportionality obtained from our numerical simulations, which yield $\delta P_{\mathrm{ch}} \approx 6 \mathrm{~Pa}$, is much smaller than $\delta P_{\mathrm{p}}$.

Let us now consider the effects of size reduction. In order to fix ideas, suppose that $h$ is reduced from 500 to $50 \mu \mathrm{m}$ (McQuain et al. ${ }^{17}$ used a $25-\mu \mathrm{m}$ depth chamber): the volume of the chamber is now $11 \mu \mathrm{l}$. Keeping the period unchanged, the flow rate is now $q=2 \mu \mathrm{l} \mathrm{s}^{-1}$ so as to maintain $q T / L^{2} h$ constant, according to Eq. (7). While delivering such a flow rate, peristaltic pumps can work with pipes of diameter as small as $d=0.19 \mathrm{~mm}$ (Ref. 29). Since the length of the pipes remains the same as previously, the external volume in the connecting pipes becomes $11 \mu \mathrm{l}$. The pressure drop inside the chamber can be calculated from the previous value using (14): $\delta P_{\text {ch }} \approx 600 \mathrm{~Pa}$, which can be neglected. The dominant contribution comes from the pressure drop in the pipes, which is now, using (13), $\delta P_{\mathrm{p}} \approx 2.5 \times 10^{4} \mathrm{~Pa}$, i.e., essentially unchanged. Note also that the Reynolds numbers inside the injection pipes and chamber are both decreased, following Eqs. (5) and (6). There is therefore a real possibility of greatly reducing the size of the device, using existing components.

\section{SUMMARY AND CONCLUSION}

We have studied two stirring protocols for use during hybridization in a DNA chip. The first one uses two pairs of syringes, while the second one operates using two pumps. In order to be efficient, a protocol should lead to very rapid and 
homogeneous mixing of macromolecules inside the chamber, and use as little volume of fluid as possible, so that chemical reactions can potentially take place at each probe of the chip with the same probability. Although both protocols allow efficient hybridization of the chip, the second one is clearly more efficient from all points of view: no regular islands formation, more homogeneous Poincaré sections, less total volume, larger Lyapunov exponents, and smaller total time required. Moreover, it is interesting to note that, from the qualitative look at top-view Poincaré sections, or else from the more quantitative results obtained from the percentage of domain covered, or at least from the calculation of Lyapunov exponents, all show that protocol B is very robust with variations of the period, since all the quantitative parameters were almost constant for $T \geq 3 \mathrm{~s}$. Therefore, its mixing efficiency does not depend much on the period, which makes it a very good candidate for this type of application.

Finally, since the protocols can be scaled down to smaller volumes [providing the parameter in Eq. (7) is kept constant], one can wonder if they can be easily integrated on a lab on a chip: the answer is yes for the second protocol. Indeed, different microfluidic pumps have already been proposed in the literature, and some of those are already used for lab on chips, while the integration of syringe-like systems seems much more difficult in practice.

\section{ACKNOWLEDGMENTS}

The authors would like to thank Jean-Noël Gence, Jérôme Broutin, and Vincent Dugas for many discussions, and Christian Nicot, Patrick Dutheil, Emmanuel Jondeau, Patrick Méjean, and Jean-Michel Perrin for their help in the experimental setup. The financial support of the BQR program of the École Centrale de Lyon, of the Program Biosécurité of the French MENRT and of the Délégation Générale de l'Armement, the FITT program of the Région Rhône-Alpes, and the PIR Microfluidique of the CNRS is also gratefully acknowledged.

${ }^{1}$ Nat. Genet. 32 (1s):1-80 (2002), supplement on DNA chips.

${ }^{2} \mathrm{R}$. T. Pon and S. Yu, "Linker phosphoramidite reagents for the attachment of the first nucleoside to underivatized solid-phase supports," Nucleic Acids Res. 32, 623 (2004).

${ }^{3}$ K. E. Richmond, M. Li, M. J. Rodesch, M. Patel, A. M. Love, C. Kim, L. L. Chu, N. Venkataramaian, S. F. Flickinger, and J. Kaysen, “Amplification and assembly of chip-eluted DNA (aaced) a method for highthroughput gene synthesis," Nucleic Acids Res. 32, 5011 (2004).

${ }^{4}$ C. J. Schaupp, G. Jiang, T. G. Myers, and M. A. Wilson, "Active mixing during hybridization improves the accuracy and reproducibility of microarray results," BioTechniques 38, 117 (2005).

${ }^{5}$ D. Kambhampati, P. E. Nielsen, and W. Knoll, "Investigating the kinetics of DNA-DNA and PNA-PNA interactions using surface plasmon resonance-enhanced fluorescence spectroscopy," Biosens. Bioelectron. 16, 1109 (2001).
${ }^{6}$ J. Zeng, A. Almadidy, J. Watterson, and U. J. Krull, "Interfacial hybridization kinetics of oligonucleotides immobilized onto fused silica surfaces," Sens. Actuators B 90, 68 (2003).

${ }^{7}$ A. E. Nkodo, J. M. Garnier, B. Tinland, H. Ren, C. Desruisseaux, L. C. McCormick, G. Drouin, and G. W. Slater, "Diffusion coefficient of DNA molecules during free solution electrophoresis," Electrophoresis 22, 2424 (2001).

${ }^{8}$ H. Aref, "Stirring by chaotic advection," J. Fluid Mech. 143, 1 (1984).

${ }^{9}$ S. Wiggins, Global Bifurcations and Chaos: Analytical Methods (Springer-Verlag, New York, 1988).

${ }^{10}$ J. M. Ottino, The Kinematics of Mixing: Stretching, Chaos and Transport (Cambridge University Press, New York, 1989).

${ }^{11}$ F. Raynal, F. Plaza, A. Beuf, Ph. Carrière, É. Souteyrand, J.-R. Martin, J.-P. Cloarec, and M. Cabrera, "Study of a chaotic mixing system for DNA chip hybridization chambers," Phys. Fluids 16, L63 (2004).

${ }^{12} \mathrm{~S}$. W. Jones and H. Aref, "Chaotic advection in pulsed source-sink systems," Phys. Fluids 31, 469 (1988).

${ }^{13}$ J. Evans, D. Liepmann, and A. P. Pisano, "Planar laminar mixer," in Proceedings of the IEEE International Workshop on Micro Electro Mechanical Systems (IEEE, New York, 1997), pp. 96-101.

${ }^{14}$ M. A. Stremler, F. R. Haselton, and H. Aref, "Designing for chaos: applications of chaotic advection at the microscale," Philos. Trans. R. Soc. London, Ser. A 362, 1019 (2004).

${ }^{15}$ M. A. Stremler and B. A. Cola, "A maximum entropy approach to optimal mixing in a pulsed source-sink flow," Phys. Fluids 18, 011701 (2006).

${ }^{16}$ B. A. Cola, D. K. Schaffer, T. S. Fisher, and M. A. Stremler, "A pulsed source-sink fluid mixing device," J. Microelectromech. Syst. 15, 259 (2006).

${ }^{17}$ M. K. McQuain, K. Seale, J. Peek, T. S. Fisher, S. Levy, M. A. Stremler, and F. Haselton, "Chaotic mixer improves microarray hybridization," Anal. Biochem. 325, 215 (2004).

${ }^{18}$ N. B. Adey, M. Lei, M. T. Howard, J. D. Jensen, D. A. Mayo, D. L. Butel, S. C. Coffin, T. C. Moyer, D. E. Slade, M. K. Spute, A. M. Hancock, G. T. Eisenhoffer, B. K. Dalley, and M. R. McNeely, "Gains in sensitivity with a device that mixes microarray hybridization solution in a $25-\mu \mathrm{m}$-thick chamber," Anal. Chem. 74, 6413 (2002).

${ }^{19}$ R. H. Liu, R. Lenigk, R. L. Druyor-Sanchez, J. Yang, and P. Grodzinski, "Hybridization enhancement using cavitation microstreaming," Anal. Chem. 75, 1911 (2003).

${ }^{20}$ V. Girault and P. A. Raviart, Finite Element Methods for Navier-Stokes Equations (Springer-Verlag, New York, 1986).

${ }^{21} \mathrm{Ph}$. Carrière, "Lyapunov spectrum determination from the FEM simulation of a chaotic advecting flow," Int. J. Numer. Methods Fluids 50, 555 (2006).

${ }^{22}$ J. P. Eckmann and D. Ruelle, "Ergodic theory of chaos," Rev. Mod. Phys. 57, 617 (1985)

${ }^{23}$ M. A. Lichtenberg and A. J. Lieberman, Regular and Chaotic Dynamics (Springer, New York, 1992).

${ }^{24}$ K. Bajer and H. K. Moffatt, "On a class of steady Stokes flows with chaotic streamlines,” J. Fluid Mech. 212, 337 (1990).

${ }^{25}$ F. Raynal and J. N. Gence, "Efficient stirring in planar, time-periodic laminar flows," Chem. Eng. Sci. 50, 631 (1995).

${ }^{26}$ V. Rom-Kedar, A. Leonard, and S. Wiggins, "An analytical study of the transport, mixing and chaos in an unsteady vortical flow," J. Fluid Mech. 214, 347 (1990).

${ }^{27}$ V. Rom-Kedar and A. C. Poje, "Universal properties of chaotic transport in the presence of diffusion," Phys. Fluids 11, 2044 (1999).

${ }^{28}$ H. A. Stone, A. D. Stroock, and A. Ajdari, "Engineering flows in small devices: Microfluidics toward lab-on-a-chip," Annu. Rev. Fluid Mech. 36, 381 (2004).

${ }^{29}$ As a reference for commercial pumps, the Masterflex ${ }^{\mathrm{TM}}$ reference N5100F and N55125 peristaltic mini pumps are considered. 